\title{
Dispersion Performance of Polycarboxylate Terpolymers with Different Alkyl Side-Chain Lengths in Pesticide Suspension Concentrate
}

\author{
Xiaodong Yang, ${ }^{1}$ Tianrui Ren $\mathbb{D}^{2},{ }^{2}$ and Bo Zhang $\mathbb{D}^{2}$ \\ ${ }^{1}$ State Key Laboratory Breeding Base of Green Pesticide and Agricultural Bioengineering/Key Laboratory of Green \\ Pesticide and Agricultural Bioengineering, Ministry of Education, Guizhou University, Guiyang 550025, China \\ ${ }^{2}$ Shanghai Engineering Research Center of Green Energy Chemical Engineering, \\ The Key Laboratory of Resource Chemistry of Ministry of Education, College of Chemistry and Materials Science, \\ Shanghai Normal University, 100 Guilin Road, Shanghai 200234, China
}

Correspondence should be addressed to Tianrui Ren; trren@shnu.edu.cn and Bo Zhang; zb830216@shnu.edu.cn

Received 23 May 2021; Accepted 9 August 2021; Published 18 August 2021

Academic Editor: Fernanda Casciatori

Copyright (c) 2021 Xiaodong Yang et al. This is an open access article distributed under the Creative Commons Attribution License, which permits unrestricted use, distribution, and reproduction in any medium, provided the original work is properly cited.

\begin{abstract}
A versatile dispersant plays a critical role in the suspension stability of pesticide suspension concentrate (SC) systems. Herein, a series of novel acrylate ester-based polycarboxylate terpolymers (PTs) were designed as dispersant for pesticide suspension concentrate and successfully synthesized in aqueous solutions from the copolymerization of $\alpha$-methacrylic acid, allyl polyoxyethylene ether, and acrylate (methyl acrylate, ethyl acrylate, and $n$-butyl acrylate). Terpolymers were characterized by FT-IR and ${ }^{1} \mathrm{H}-\mathrm{NMR}$ to confirm their structure. The surface activities of PTs were evaluated according to critical micelle concentration (CMC) and the surface tension at CMC $\left(\gamma_{\mathrm{CMC}}\right)$. PT-3 with the longest alkyl side-chain length displayed the most superior surface activity due to its lowest CMC value compared with that of the other PTs. Additionally, the dispersion properties of PTs as dispersants in a $600 \mathrm{~g} / \mathrm{L}$ imidacloprid SC were evaluated. Results showed that compared to the other two PTs, PT-3 contributes to the excellent dispersion properties on imidacloprid SC. Meanwhile, the obtained SC system exhibited shear thinning behavior under high-speed shearing showing typical features of pseudo-plastic non-Newtonian fluids, which conforms to the Herschel-Buckley model. Our results revealed that the effect of the alkyl side-chain length of dispersants could be considered the primary modulator of dispersion performance of SCs.
\end{abstract}

\section{Introduction}

Pesticide aqueous SC, as an eco-friendly water-based formulation, exhibits excellent advantages, such as high suspension and bioefficacy, low cost, and safety for operators $[1,2]$. However, SC belongs to a thermodynamic and unstable dispersed system and easily encounters physical stability obstacles, such as Ostwald ripening and the flocculation or agglomeration of particles [3], which encloses lots of water and results in increasing the viscosity and decreasing the fluidity of SC. This is especially true for high-concentration SC. Ionic polymeric surfactants, which adsorbed onto solid particles and prevent the particles from aggregation because of electrostatic or steric repulsion, were often served as dispersants to address the above problems [4, 5]. Among these polymers, polyacrylic acid (PAA) is most widely used as a dispersant. Compared to PAA, the molecular structure of polycarboxylate copolymers is easy to be modified, which can meet specific properties for various applications.

Especially, polycarboxylate copolymers such as terpolymers are considered as a versatile dispersant because of their numerous branched chain groups, including carboxyl group, sulfonic acid group, amino group, and polyoxyethylene group side chains, which is widely used in concrete, ceramics, paints, and dye to obviously 
improve suspension stability of the aqueous suspension system [6-9]. This is due to the fact that the carboxylate groups of the admixtures act as anchors to adsorb on the surface of the particles and show electrostatic effect. Meanwhile, the polyethylene oxide side chains also prevent the steric effect and prevent agglomeration of the particles [10-13]. For example, Wang et al. and Bai et al. found that the particular molecular structure of polycarboxylate-based terpolymers guaranteed their good dispersibility for $\mathrm{CaCO}_{3}$ suspensions and had strong antiredeposition power $[14,15]$. Hence, it has great significance in designing suitable polycarboxylate terpolymers as dispersants for efficiently improving the stability of pesticide SC.

Imidacloprid is a chloro-nicotinyl insecticide which specifically blocks the microtinergic neuronal pathway, which is commonly used in rice, soyabean, maize, potatoes, vegetables, sugar beets, fruit, cotton, hops, and turf and is especially systemic when used as the seed or soil treatment [16]. Herein, three kinds of polycarboxylate terpolymers with different carbon side-chain lengths as an anchoring unit were designed and successfully synthesized. Moreover, the obtained PTs were selected as dispersants to prepare $600 \mathrm{~g} / \mathrm{L}$ imidacloprid SC to verify its effect on the suspension stability of SC.

\section{Experimental Section}

2.1. Materials. Technical-grade imidacloprid (98.5\% purity) was kindly supplied by Jiangsu Jiannong Agrochemical and Chemical Co. Ltd. (China). Allyl polyoxyethylene ether (APEG), $\alpha$-methacrylic acid (MAA), methyl acrylate (MA), ethyl acrylate (EA), and $n$-butyl acrylate (BA) were obtained from Sinopharm Chemical Regent Co. (China). Xanthan gum was of food reagent grade and obtained from the Shanghai Ji Hu Chemical Co., Ltd of China. The SR-08 wetting agent was purchased from the Shanghai ShiDa Micromolecular Materials Co., Ltd. The ethylene glycol antifreeze agent was obtained from Shanghai Titan Technology Co., Ltd. Double distilled water was used in the experiment. All chemicals were of analytic grade and were used without further purification.

\subsection{Synthesis of Polycarboxylate Terpolymers (PTs).} Scheme 1 illustrates the synthesis of PTs by free-radical polymerization of APEG and MAA with MA, EA, and BA, which are denoted as PT-1, PT-2, and PT-3, respectively. The mole ratio of APEG: MAA: MA/EA/BA was $4: 1: 1$. Specifically, 0.5 wt. $\%$ of initiator and 1 wt. $\%$ chain transfer agent were added to the monomers. Appropriate amounts of distilled water and sodium bisulfite (a certain amount of chain transfer agent, water, and methanol) were added into a $250 \mathrm{~mL}$ four-mouth flask and stirred at $80^{\circ} \mathrm{C}$. APEG, MAA, and $\mathrm{MA} / \mathrm{EA} / \mathrm{BA}$ were dissolved in methanol and added dropwise into the reactor. Then, the mixture was allowed to react at $80^{\circ} \mathrm{C}$ for $3.5 \mathrm{~h}$. Next, the mixture was cooled to room temperature and its $\mathrm{pH}$ was adjusted to $7-8$ with $30 \mathrm{wt} . \%$ $\mathrm{NaOH}$. Finally, the PTs were obtained.
2.3. Structural Characterization of PTs. The PTs were purified with a dialysis bag and the powder was obtained by freezedrying. Infrared spectroscopy was analyzed by potassium bromide pellet method. Using deuterium instead of water as the solvent, the structure was characterized by ${ }^{1} \mathrm{H}$ NMR spectroscopy.

\subsection{Measurement of Critical Micelle Concentration (CMC)} Values of PTs. Surface tension of PT aqueous solutions was measured at $25^{\circ} \mathrm{C}$ with a JYW-2008 tensiometer (CDTM Ltd., China) by the Wilhelmy plate method. Prior to each experiment, the instrument was calibrated and checked by measuring the surface tension of distilled water. The CMC value was obtained from the breakpoint of the plot of surface tension $(\gamma)$ versus $\ln c$ [17].

According to Gibbs law, the maximum surface excess concentration $\left(\Gamma_{\mathrm{CMC}}\right)$ and the minimum surface area per surfactant molecule $\left(A_{\mathrm{CMC}}\right)$ were calculated by the Gibbs adsorption isotherm equations (equations (1) and (2)). And, the Gibbs free energy of micellization, $\Delta G_{\text {mic }}^{\theta}$, and the Gibbs free energy of adsorption $\Delta G_{\text {ad }}^{\theta}$ were calculated using equations (3) and (4) [4].

$$
\begin{aligned}
\Gamma_{\mathrm{CMC}} & =\frac{-1}{2.303 n T R}\left(\frac{\partial \gamma}{\partial \lg C}\right)_{T}, \\
A_{\mathrm{cmc}} & =\frac{1}{N_{A} \Gamma_{\mathrm{cmc}}}, \\
\Delta G_{\mathrm{mic}}^{\Theta} & =n R T \ln \mathrm{CMC}, \\
\Delta G_{\mathrm{ad}}^{\Theta} & =n R T \ln \mathrm{CMC}-0.6023\left(\gamma_{0}-\gamma_{\mathrm{cmc}}\right) A_{\mathrm{cmc}},
\end{aligned}
$$

where $\gamma_{0}, N_{A}$, and $\gamma_{\mathrm{CMC}}$ are the PTs of pure water, Avogadro's number, and the surfactant solutions at CMC, respectively. Here, $n$ is the number of species at the interface whose concentration changes with surfactant concentration ( $n=1$ for a gemini surfactant and $n=2$ for an anionic surfactant). $R$ and $T$ take their usual meanings.

2.5. Preparation of $600 \mathrm{~g} / \mathrm{L}$ Imidacloprid SC. $600 \mathrm{~g} / \mathrm{L}$ imidacloprid SCs were prepared by wet sand grinding technology. The optimum formulation of SCs was determined by preexperiment. Typically, $50 \mathrm{~g}$ imidacloprid, $2 \mathrm{~g}$ PTs, $1 \mathrm{~g}$ wetting agent SR-08, $4 \mathrm{~g}$ xanthan gum, and $4 \mathrm{~g}$ ethylene glycol were mixed together with the rest of the water. Then, the mixture was obtained after grinding under room temperature until the particle size distribution of less than $3 \mu \mathrm{m}$.

\subsection{Characterization of the $600 \mathrm{~g} / \mathrm{L}$ Imidacloprid SC}

2.6.1. Zeta Potential of $600 \mathrm{~g} / \mathrm{L}$ Imidacloprid SC. A small amount of $600 \mathrm{~g} / \mathrm{L}$ imidacloprid SC was weighed in a $250 \mathrm{~mL}$ beaker using an analytical balance. After diluting the imidacloprid to a given concentration by adding deionized water, the solution was transferred to a $50 \mathrm{~mL}$ centrifuge tube and centrifuged at $3000 \mathrm{r} / \mathrm{min}$ for $5 \mathrm{~min}$. The zeta 


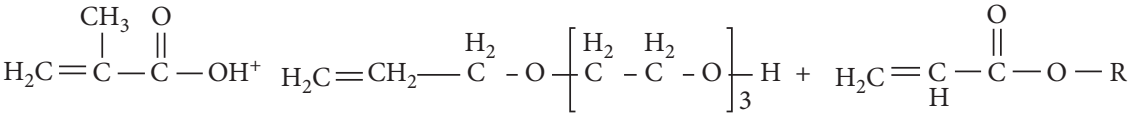

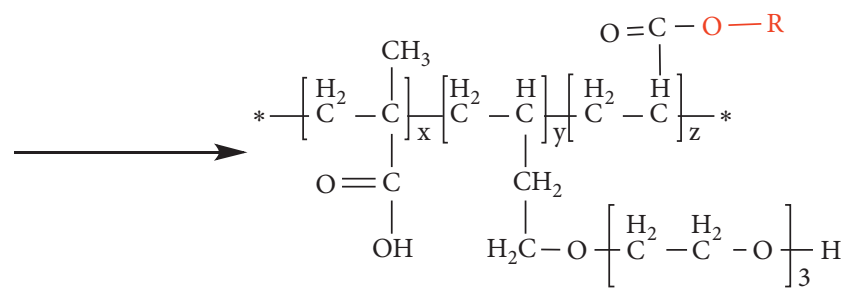

Scheme 1: Synthesis process of PTs.

potential of the supernatant of the centrifugation was measured on a Malvern Nano ZS90. Each sample was measured three times, and the average was used.

\subsection{Measurement of Suspensibility of $600 \mathrm{~g} / \mathrm{L}$ Imidacloprid SC}

2.7.1. Suspensibility of $600 \mathrm{~g} / \mathrm{L}$ Imidacloprid SC. The suspensibility of SC was measured according to GB/T 148252006.

2.7.2. Determination of SC Viscosity and Rheological Properties. The viscosity and rheological properties of the SC were measured using an Anton Par MCR 102 rotational rheometer. The shear viscosity of SC samples was studied at $25^{\circ} \mathrm{C}$ with a shear rate of $0-1000 \mathrm{~s}^{-1}$.

Viscosity recovery as a critical factor of SC was measured in three stages. That is, the sample was subjected to a constant low-speed shear $\left(0.25 \mathrm{~s}^{-1}\right)$ in the initial $25 \mathrm{~s}$, highspeed shear $\left(1000 \mathrm{~s}^{-1}\right)$ in the next $10 \mathrm{~s}$, and then constant low-speed shear $\left(0.25 \mathrm{~s}^{-1}\right)$ for recovery.

\section{Results and Discussion}

The chemical structures of PTs were analyzed by FT-IR and ${ }^{1} \mathrm{H}-\mathrm{NMR}$ (Figure 1). Taking the case of PT-3, as presented in Figure 1(a), the wide stretching vibration peak of $-\mathrm{OH}$ is at $3394 \mathrm{~cm}^{-1}$, the stretching vibration peaks of methyl and methylene appear at $2922 \mathrm{~cm}^{-1}$ and $2871 \mathrm{~cm}^{-1}$, and the characteristic peak of $\mathrm{C}=\mathrm{O}$ locates at $1735 \mathrm{~cm}^{-1}$. A value of $1453 \mathrm{~cm}^{-1}$ is the bending vibration of methyl and methylene, $1109 \mathrm{~cm}^{-1}$ is the stretching vibration peak of C-O-C, and the characteristic peaks of double bond at $2500-2000 \mathrm{~cm}^{-1}$ disappear after copolymerization.

Figure 1(b) shows ${ }^{1} \mathrm{H}-\mathrm{NMR}$ spectrum of PT-3. Among them, $\delta=4.70$ is the solvent peak and $\delta=3.8-3.47$ is the oxyethylene structural unit $\left(\mathrm{CH}_{2} \mathrm{CH}_{2} \mathrm{O}\right)$ in the polyether. The proton peak of $\mathrm{H}$ on the main carbon chain is at $\delta=1.36-1.58$, and the proton peaks of $-\mathrm{CH}_{3}$ and $-\mathrm{CH}_{2}$ are near $\delta=0.9$. After $\delta=5.7$, there is no double bond proton peak, indicating that it disappears and participates in the reaction polymerization.

Furthermore, the GPC analysis was utilized to obtain the molecular weight of various PTs and their distributions. The
$M_{n}$ and $M_{w} / M_{n}$ of PTs are listed in Table 1 . The $M_{n}$ and $M_{w} / M_{n}$ of PT-1, PT-2, and PT-3 are 2160, 2310, and 2287, and $2.47,2.83$, and 2.28 , respectively. The results indicate that the various dispersants are randomly copolymerized by free-radical copolymerization.

3.1. CMC and Thermodynamic Properties. CMC and surface tension at this concentration $\left(\gamma_{\mathrm{CMC}}\right)$ are important factors to measure the surface activity of surfactant aqueous solutions. Figure 2 and Table 2 show the surface tension of PTs, from which we realize that the aqueous solution exhibited a continuous decrease of surface tension and remained unchanged with the concentration increasing to CMCs. Compared with other PTs, the CMC and $\gamma_{\mathrm{CMC}}$ values of PT3 are minimum, which indicated that PT-3 has the most superior surface activity. The hydrophobic monomer BA makes PT-3 more hydrophobic, resulting in its lower CMC and $\gamma_{\mathrm{CMC}}[18]$.

The adsorption data of PTs are listed in Table 2. PT-3 has a largest $\Gamma_{\max }$ and smallest $A_{\min }$, conferring its superior surface activity. Meanwhile, the negative values of $\Delta G_{\text {mic }}$ show the micellization process, and it is a thermodynamic spontaneous process. The order of absolute value of $\Delta G_{\text {mic }}$ is PT-3 > PT-2>PT-1, which suggests that PT-3 has the strongest driving force for micellization or adsorption at the air-liquid interface [19]. This could be attributed to the greatest hydrophobic performances of PT-3, leading to an easier formation of micelles [20].

3.2. Impacts of Dispersant Dosage on Zeta Potential and Viscosity of $600 \mathrm{~g} / \mathrm{L}$ Imidacloprid SC. Zeta potential is one of the key parameters usually utilized to estimate suspension stability, which reflects the effective adsorption of dispersants on the surface of particles. Figure 3(a) shows the effects of PTs on the zeta potential of $600 \mathrm{~g} / \mathrm{L}$ imidacloprid SC. It was found that the amount of PTs dramatically affected the zeta potential. The zeta potential decreases sharply with increasing PTs from 0 to $2 \mathrm{wt} . \%$. Moreover, the continued increase of the dispersant beyond $2 \mathrm{wt} . \%$ up to $6 \mathrm{wt} . \%$ decreases a little zeta potential, suggesting that the dispersants gradually reached saturation adsorption on the surface of pesticide particles at the concentration of $6 \mathrm{wt} . \%$. Surprisingly, the zeta potential decreases with further increase in PT 


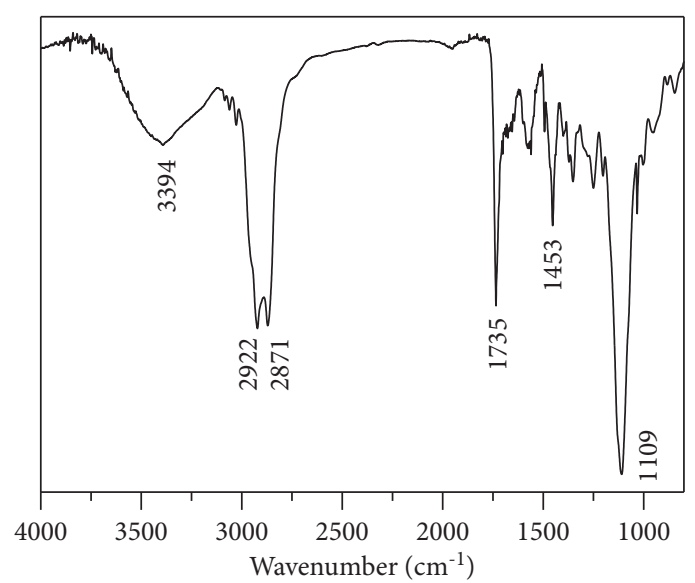

(a)

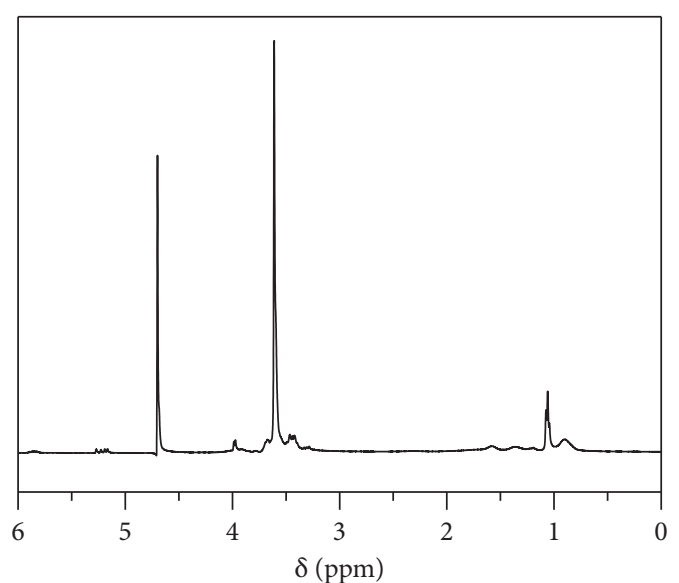

(b)

Figure 1: FTIR spectrum and ${ }^{1} \mathrm{H}-\mathrm{NMR}$ spectra of PT-3.

TABle 1: Monomer combination and GPC results for the various PTs.

\begin{tabular}{lcccccc}
\hline Sample & \multicolumn{2}{c}{ Monomer } & \multicolumn{2}{c}{ Ratio } & BA & $M_{n}(\mathrm{~g} / \mathrm{mol})$ \\
& MAA & APEG & MA & EA & BA & 2160 \\
PT-1 & 1 & 4 & 1 & & & 2.47 \\
PT-2 & 1 & 4 & & 1 & 1 & 2.83 \\
PT-3 & 1 & 4 & & & 1 & 2.287 \\
\hline
\end{tabular}

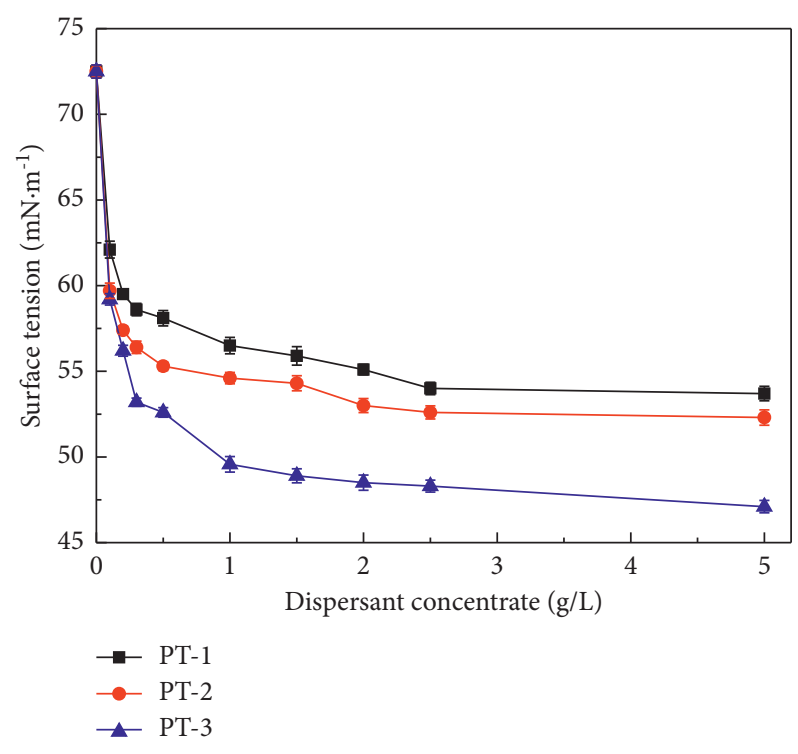

Figure 2: Evaluation of the CMC values of PTs by means of the Wilhelmy plate method.

TABLE 2: Surface properties of PTs.

\begin{tabular}{lccccc}
\hline Dispersants & $\gamma_{\mathrm{CMC}}(\mathrm{mN} / \mathrm{m})$ & $\mathrm{CMC}(\mathrm{g} / \mathrm{L})$ & $\Gamma_{\max }\left(\times 10^{-6} \mathrm{~mol} / \mathrm{m}^{2}\right)$ & $A_{\mathrm{CMC}}(\mathrm{nm} / \mathrm{molecule})$ & $\Delta G_{\mathrm{mic}}(\mathrm{kJ} / \mathrm{mol})$ \\
\hline PT-1 & 53.70 & 2.50 & 4.66 & 0.354 & -20.728 \\
PT-2 & 52.30 & 2.15 & 5.36 & 0.310 & -22.854 \\
PT-3 & 47.10 & 1.33 & 6.58 & 0.252 & -24.498 \\
\hline
\end{tabular}

dosage. This is because that excessive counterions of dispersants enter into the diffusion layer in the double electrical layer, resulting in the slight decrease in zeta potential values
[21]. Additionally, the absolute of zeta potential of $600 \mathrm{~g} / \mathrm{L}$ imidacloprid SC obtained from PT-3 is minimum, indicating that PT-3 could provide stronger electrostatic 


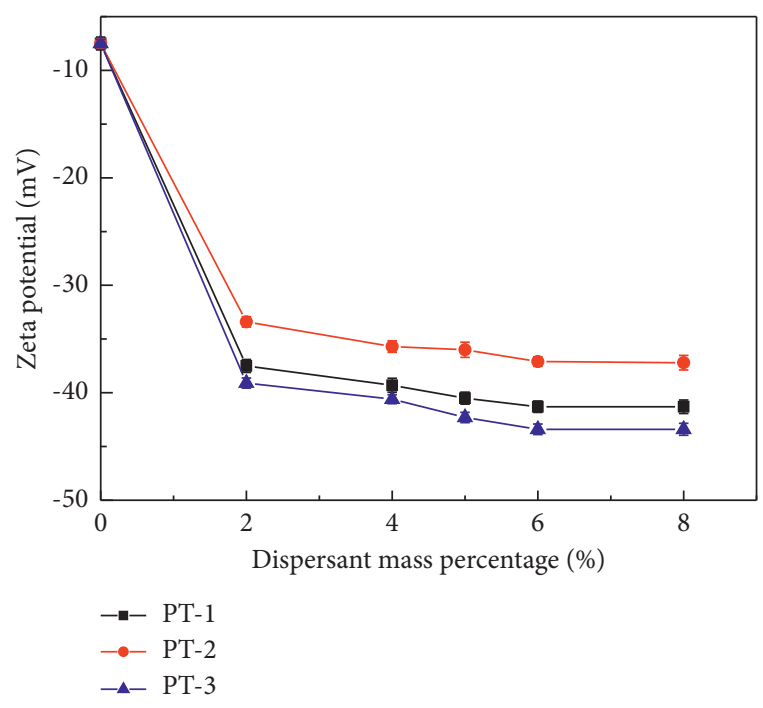

(a)

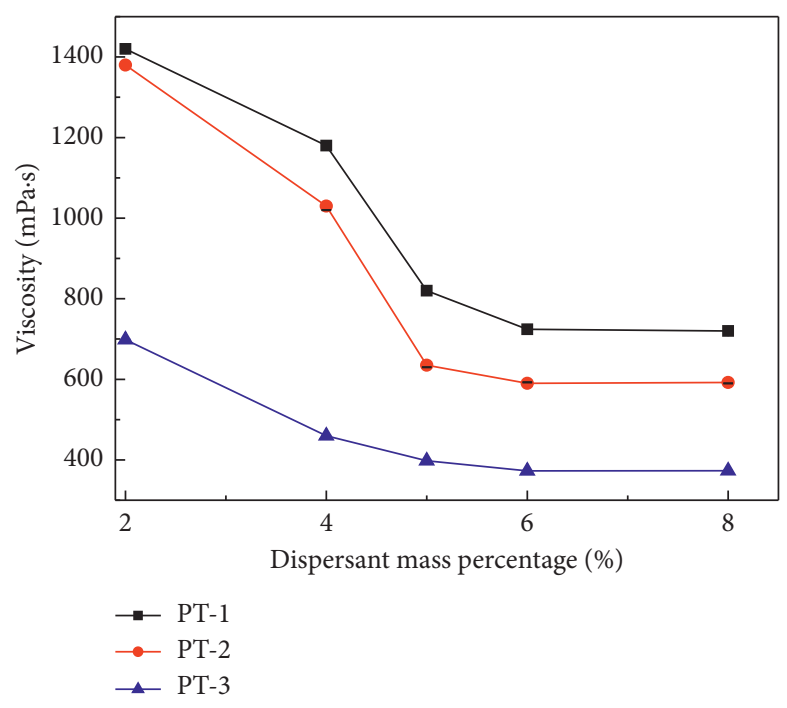

(b)

Figure 3: Impacts of PTs on zeta potential and viscosity of $600 \mathrm{~g} / \mathrm{L}$ imidacloprid SC.

repulsion than PT-1 and PT-2. Hence, the optimal dispersant and additive amount are PT-3 and 6\%, respectively.

The dispersant can effectively improve the viscosity of suspension systems and further effect on suspension stability. Subsequently, we investigated the effect of PT amounts on the viscosity of $600 \mathrm{~g} / \mathrm{L}$ imidacloprid SC. As illustrated in Figure 3(b), it was found that the viscosity of imidacloprid SC sharply decreased and then slightly increased with increasing amounts of the PTs. When the dispersant dosage reached $6 \%$, the viscosity of the SC is minimum, which agrees with the results of zeta potential (Figure 3(a)). However, when the amount of dispersants is above $6 \%$, it leads to a slight increase in viscosity, which could be because that the excess dispersant causes counterions to enter the diffusion layer and the electrostatic repulsion decreases. Therefore, the combined bridging and depletion effects result in increased viscosity of the suspension system [19]. Notably, PT-3 reveals a greater influence on the viscosity of the SC, indicating that PT-3 exhibits superior dispersion capacity for SC.

We further investigated the thermal stability and rheological properties of $600 \mathrm{~g} / \mathrm{L}$ imidacloprid SC made of PTs in 6 wt.\% dosage.

3.3. Analysis of Thermal Stability of $600 \mathrm{~g} / \mathrm{L}$ Imidacloprid SC. The physical stability of pesticide SC systems is of great significance for its long-term storage. The long-term storage stability is very important for pesticide formulations for practical usage in agriculture since delamination, caking, and degradation of the active pesticide may occur during storage. To study storage stability, the optimum SC samples made of PTs in 6 wt.\% dosage were kept under $54 \pm 2^{\circ} \mathrm{C}$ for 14 days to determine their thermal stability (Table 3 ).

The changes of suspension rate and particle size distribution are important quality indexes and often used to characterize the physical stability of SC. As listed in Table 3, the suspension rate of the SC sample made of PT-3 is still greater than $95 \%$, while the average diameter of particles is about 2.24 and $5.86 \mu \mathrm{m}$, respectively, before and after thermal storage, indicating PT-3 can effectively improve the physical stability of $600 \mathrm{~g} / \mathrm{L}$ imidacloprid SC. Moreover, the SC prepared from PT-3 has suitable viscosity and viscosity change after thermal storage. Accordingly, the PT-3 is a promising dispersant for improving the thermal stability of $600 \mathrm{~g} / \mathrm{L}$ imidacloprid SC.

\subsection{Analysis of Rheological Properties of $600 \mathrm{~g} / \mathrm{L}$ Imidacloprid} $S C$. The suspension stability of SC systems was investigated by the rheological method. Apparent viscosity and thixotropy can obviously reflect the suspension stability of the SC system $[22,23]$. Figure 4 shows the rheological curves of the PTs with $600 \mathrm{~g} / \mathrm{L}$ imidacloprid SC in $6 \mathrm{wt} . \%$ dosage. The apparent viscosity of SC systems decreases with increasing shear rate, suggesting that the SC systems exhibited shear thinning behavior. Thus, three SCs had the characteristics of typical non-Newtonian pseudo-plastic fluid.

The three-stage thixotropy was used to verify the viscosity recovery of SC systems and can reflect the relationship between the viscosity and the change of network structure of the SC system. The results of the three-step thixotropy test are shown in the illustration of Figure 4(b). At low-speed shear, the spatial network structure of the SCs is stable and the systems exhibit high viscosity. Moreover, when the shear rate reached $1000 \mathrm{~s}^{-1}$, the structures of SC systems were damaged, resulting in a rapid decline in viscosity. In the third stage $\left(0.25 \mathrm{~s}^{-1}\right.$ after $\left.35 \mathrm{~s}\right)$, the structure of the SC system was restored. Notably, SC obtained from PT-3 had a higher structural recovery than SCs made of PT-1 and PT-2, indicating that SC made of PT-3 had higher stability, which is in accordance with the results of thermal stability (Table 3 ). 
TABLE 3: The performances of $600 \mathrm{~g} / \mathrm{L}$ imidacloprid SC before $(A)$ and after $(B)$ thermal storage.

\begin{tabular}{lcccccc}
\hline \multirow{2}{*}{ Sample } & \multicolumn{2}{c}{ Viscosity $(\mathrm{MPa} \cdot \mathrm{s})$} & \multicolumn{2}{c}{ Suspension rate $(\%)$} & \multicolumn{2}{c}{ Particles size $(\mu \mathrm{m})$} \\
& $A$ & $B$ & $A$ & $92.8 \pm 0.3$ & $89.4 \pm 0.1$ & $A$ \\
\hline PT-1 & 724 & 1265 & $94.6 \pm 0.5$ & $90.2 \pm 0.2$ & 2.13 & 2.17 \\
PT-2 & 590 & 972 & $96.3 \pm 0.5$ & $95.6 \pm 0.3$ & 2.33 \\
PT-3 & 373.4 & 525 & 2.24 & 5.86 \\
\hline
\end{tabular}

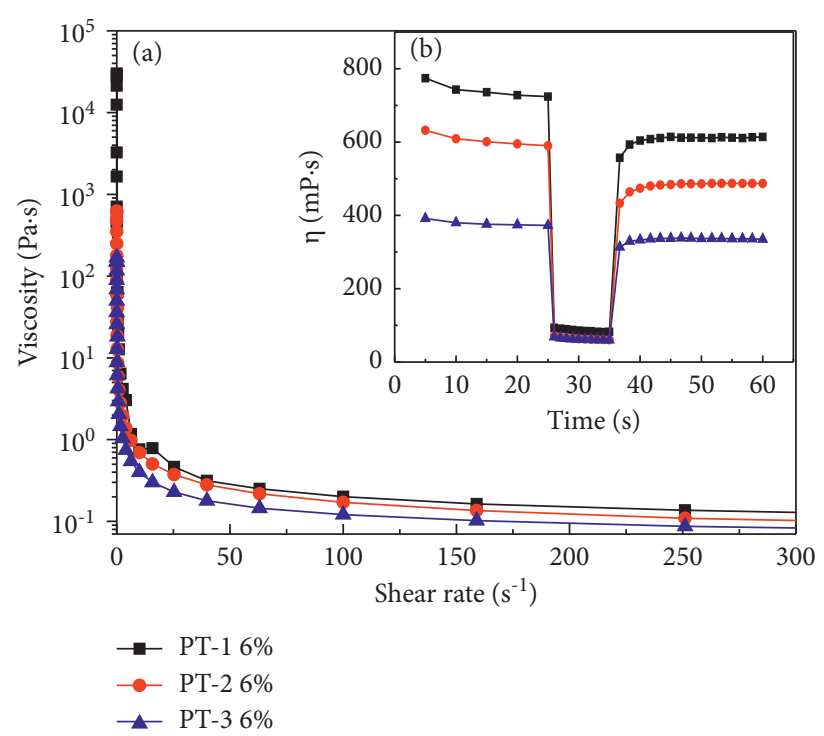

FIgURE 4: Rheological properties (a) and three-stage shearing curve (b) of $600 \mathrm{~g} / \mathrm{L}$ imidacloprid SC.

3.5. The Proposed Stability Mechanism of Imidacloprid SC Prepared from PTs. When the pesticide particles were dispersed into aqueous solution, the particles were first wetted by water, and by increasing the wettability of the pesticide surface, dispersants reduce the frequently observed tendency of hydrophobic pesticide particles to spontaneously aggregate in aqueous suspensions; thus, the lower the surface tension of the dispersant aqueous solutions is, the easier the wetting of pesticide particles will be so as to contribute to the dispersion of pesticide particles more readily into aqueous solution $[4,24]$. Compared with PT-1 and PT-2, PT-3 had the lowest CMC (Table 2). Therefore, PT-3 exhibits much better strong adsorption and wetting ability at the pesticide surface.

Remarkably, PT-3 has carboxylate groups and the longest hydrophobic alkyl side chain, as the anchoring points, which make it steadily be adsorbed on the surface imidacloprid particles. Meanwhile, PTs contain neutral EO pendants, which provide steric hindrance among imidacloprid particles. For the above reasons, PT-3 is endowed with a superior ability to stabilize and disperse imidacloprid particles and maintained the suspension stability of imidacloprid SC.

\section{Conclusion}

Three polycarboxylate terpolymers containing different carbon side-chain lengths were successfully synthesized by freeradical polymerization. PT-3 serves as an effective dispersant to stabilize the $600 \mathrm{~g} / \mathrm{L}$ imidacloprid SC. When PT-3 dosage reached $6 \%$, the SC had the minimum viscosity and the maximum zeta potential, which are very beneficial for improving its suspension stability. Meanwhile, rheological properties showed further evidence that the SC made of PT-3 exhibits excellent suspension stability compared to other PTs.

\section{Data Availability}

The data used to support the findings of this study are included within the article.

\section{Conflicts of Interest}

The authors declare that they have no conflicts of interest.

\section{Acknowledgments}

The authors thank the Chinese Characters of Shanghai Agricultural Sciences (2018) (No. 3-3).

\section{References}

[1] R. Peng, Y. Pang, X. Qiu, Y. Qian, and M. Zhou, "Synthesis of anti-photolysis lignin-based dispersant and its application in pesticide suspension concentrate," RSC Advances, vol. 10, no. 23, pp. 13830-13837, 2020.

[2] X. Qiu, W. Zeng, W. Yu et al., "Alkyl chain cross-linked sulfobutylated lignosulfonate: a highly efficient dispersant for carbendazim suspension concentrate," ACS Sustainable Chemistry \& Engineering, vol. 3, no. 7, pp. 1551-1557, 2015. 
[3] T. Tadros, P. Izquierdo, J. Esquena, and C. Solans, "Formation and stability of nano-emulsions," Advances in Colloid and Interface Science, vol. 108-109, pp. 303-318, 2004.

[4] L. Zhang, X. Guo, B. Zhang, and T. Ren, "Synthesis of nonylphenol polyoxyethylene oligomer and application as an effective dispersant in pyraclostrobin suspension concentrate," Designed Monomers and Polymers, vol. 22, no. 1, pp. 122-129, 2019.

[5] S. Haas, H.-W. Hässlin, and C. Schlatter, "Influence of polymeric surfactants on pesticidal suspension concentrates: dispersing ability, milling efficiency and stabilization power," Colloids and Surfaces A: Physicochemical and Engineering Aspects, vol. 183-185, pp. 785-793, 2001.

[6] K. Amini, A. Ghasemi, S. Soleimani Amiri, S. Mirvalad, and A. Habibnejad Korayem, "The synergic effects of metakaolin and polycarboxylate-ether on dispersion of graphene oxide in cementitious environments and macro-level properties of graphene oxide modified cement composites," Construction and Building Materials, vol. 270, Article ID 121462, 2021.

[7] Y. Li, Y. Zhang, J. Zheng et al., "Dispersion and rheological properties of concentrated kaolin suspensions with polycarboxylate copolymers bearing comb-like side chains," Journal of the European Ceramic Society, vol. 34, no. 1, pp. 137-146, 2014.

[8] F. Najafi, Z. Ranjbar, B. Shirkavand Hadavand, and S. Montazeri, "Synthesis and characterization of comb polycarboxylic acid dispersants for coatings," Journal of Applied Polymer Science, vol. 126, no. 3, pp. 877-881, 2012.

[9] H. Gharanjig, K. Gharanjig, and A. Khosravi, "Effects of the side chain density of polycarboxylate dispersants on dye dispersion properties," Coloration Technology, vol. 135, no. 2, pp. 160-168, 2019.

[10] X. Qiu, X. Peng, C. Yi, and Y. Deng, "Effect of side chains and sulfonic groups on the performance of polycarboxylate-type superplasticizers in concentrated cement suspensions," Journal of Dispersion Science and Technology, vol. 32, no. 2, pp. 203-212, 2011.

[11] J. Liu, Q. Ran, C. Miao, and M. Qiao, "Effects of grafting densities of comb-like copolymer on the dispersion properties of concentrated cement suspensions," Materials Transactions, vol. 53, no. 3, pp. 553-558, 2012.

[12] C.-Z. Li, N.-Q. Feng, Y.-D. Li, and R.-J. Chen, "Effects of polyethlene oxide chains on the performance of polycarboxylate-type water-reducers," Cement and Concrete Research, vol. 35, no. 5, pp. 867-873, 2005.

[13] B. Felekoglu and H. Sarıkahya, "Effect of chemical structure of polycarboxylate-based superplasticizers on workability retention of self-compacting concrete," Construction and Building Materials, vol. 22, no. 9, pp. 1972-1980, 2008.

[14] G. Wang, Y. Bai, X. Ma, W. Wang, Q. Yin, and Z. Du, "Effects of the PEG length of polycarboxylate-based terpolymers on their dispersion properties," Journal of Molecular Liquids, vol. 225, pp. 333-338, 2017.

[15] Y. Bai, X. Ma, W. Wang, Q. Yin, Z. Du, and G. Wang, "Synthesis, aggregation and dispersity properties of novels amphiphilic comb-like terpolymers," Colloids and Surfaces A: Physicochemical and Engineering Aspects, vol. 526, pp. 40-47, 2017.

[16] K. A. Sumon, A. K. Ritika, E. T. H. M. Peeters et al., "Effects of imidacloprid on the ecology of sub-tropical freshwater microcosms," Environmental Pollution, vol. 236, pp. 432-441, 2018.

[17] W. Zhang, G. J. Dong, H. Yang, J. Sun, J. Zhou, and J. Wang, "Synthesis, surface and aggregation properties of a series of amphiphilic dendritic copolymers," Colloids and Surfaces A: Physicochemical and Engineering Aspects, vol. 348, no. 1-3, pp. 45-48, 2009.

[18] R. Hoogenboom, D. Popescu, W. Steinhauer, H. Keul, and M. Möller, "Nitroxide-mediated copolymerization of 2hydroxyethyl acrylate and 2-hydroxypropyl acrylate: copolymerization kinetics and thermoresponsive properties," Macromolecular Rapid Communications, vol. 30, no. 23, pp. 2042-2048, 2009.

[19] C. Ren, F. Wang, Z. Zhang, H. Nie, N. Li, and M. Cui, "Synthesis, surface activity and aggregation behavior of gemini imidazolium surfactants 1, 3-bis (3-alkylimidazolium1-yl) propane bromide," Colloids and Surfaces A: Physicochemical and Engineering Aspects, vol. 467, pp. 1-8, 2015.

[20] P. Raffa, D. A. Z. Wever, F. Picchioni, and A. A. Broekhuis, "Polymeric surfactants: synthesis, properties, and links to applications," Chemical Reviews, vol. 115, no. 16, pp. 8504-8563, 2015.

[21] I. Grillo and J. Penfold, "Self-assembly of mixed anionic and nonionic surfactants in aqueous solution," Langmuir, vol. 27, no. 12, pp. 7453-7463, 2011.

[22] B. A. Moreira, F. de Oliveira Arouca, and J. J. R. Damasceno, "Analysis of suspension sedimentation in fluids with rheological shear-thinning properties and thixotropic effects," Powder Technology, vol. 308, pp. 290-297, 2017.

[23] M. A. Faers and G. R. Kneebone, "Application of rheological measurements for probing the sedimentation of suspension concentrate formulations," Pesticide Science, vol. 55, no. 3, pp. 312-325, 1999.

[24] D. Das, U. Dash, J. Meher, and P. K. Misra, "Improving stability of concentrated coal-water slurry using mixture of a natural and synthetic surfactants," Fuel Processing Technology, vol. 113, pp. 41-51, 2013. 